\title{
A Case of Catatonia and Neuroleptic Malignant Syndrome Probably Associated with Antipsychotic in Korea
}

\author{
Ho-Dong Choi, Kyoung-Keun Kim and Bon-Hoon Koo ${ }^{凶}$ \\ Department of Psychiatry, Yeungnam University College of Medicine, Daegu, Korea
}

Several studies have reported on catatonia caused by the use of antipsychotic drugs and on the association between catatonia and neuroleptic malignant syndrome (NMS), but none has reported such a case in Korea. Here, we report the case of a 20 -year-old woman whose catatonia and NMS appeared associated with the administration of an atypical antipsychotic drug. We discuss the association between NMS and catatonia due to neuroleptic use.

Psychiatry Investig 2011;8:174-177

Key Words Atypical antipsychotics, Catatonia, Neuroleptic malignant syndrome.

\section{INTRODUCTION}

Catatonia is a syndrome, comprised of such symptoms as motor immobility, excessive motor activity, extreme negativism, and stereotyped movements. It mainly occurs in primary mood or psychotic illness and in medical and surgical conditions such as neoplasms, encephalitis, head traumas, diabetes, and metabolic disorders. ${ }^{1}$ Since Kahlbaum coined the name "vesania katatonica" in 1874, Kraepelin began using "catatonia" for a schizophrenia subtype in 1899, and Kleist explained catatonia as a kind of extrapyramidal disorder related to the basal ganglia in $1943 .^{2}$ Recently, Kahlbaum explained catatonia as a disease, inferring this concept from various studies and experiences showed patients with catatonia symptoms commonly responded favorably to lorazepam and electroconvulsive therapy (ECT). ${ }^{3}$ However, no one has yet clearly explained the nature and neurobiological basis of catatonia. ${ }^{4}$

Neuroleptic malignant syndrome (NMS) is a rare syndrome, observed in only $0.2 \%$ of psychiatric patients, ${ }^{5}$ and patients typically show symptoms such as an altered mental state, muscle rigidity, tremor, tachycardia, hyperpyrexia, leucocytosis, and el-

Received: October 18, 2010 Revised: November 29, 2010

Accepted: December 7, 2010 Available online: January 10, 2011

$\triangle$ Correspondence: Bon-Hoon Koo, MD, PhD

Department of Psychiatry, Yeungnam University College of Medicine, 317-1 Daemyeong 5-dong, Nam-gu, Daegu 705-717, Korea

Tel: +82-53-622-3343, Fax: +82-53-629-0256, E-mail: vijnana@chol.com

(a) This is an Open Access article distributed under the terms of the Creative Commons Attribution Non-Commercial License (http://creativecommons.org/licenses/bync/3.0) which permits unrestricted non-commercial use, distribution, and reproduction in any medium, provided the original work is properly cited. evated serum creatine kinase. ${ }^{6}$ Any antipsychotic drug, including the atypical ones, can cause NMS.?

Recently, several researchers have reported studies on antipsychotic-induced catatonia and the association between catatonia and NMS, ${ }^{2,8-10}$ but none were from Korea. Here, we report a case of catatonia and NMS correlated with the administration of an atypical antipsychotic drug.

\section{CASE}

A 20-year-old female with no neuropsychiatric history gradually became restless around July 2009. She complained of anxiety, cried often, and could not sleep. Several days later, the patient experienced auditory hallucination, she screamed in her home, "I cannot kill mom! I should die!" and attempted to jump out a window. When her mother stopped the patient, she ran to the kitchen and picked up a knife. From that time on, she talked continuously to herself. As these symptoms continued, she was admitted to the psychiatric hospital with a impression of psychotic disorder not otherwise specified (NOS).

She received olanzapine $10 \mathrm{mg} /$ daily for 3 days, but her symptoms did not change, and side-effect symptoms appeared such as sedation, drooling and dysarthria. Thus, the psychiatrist switched her prescription to risperidone $4 \mathrm{mg} /$ daily. After 2 days of risperidone, the patient spent most of her time on the bed without any appropriate communication. As she began showing such behaviors as mumbling and chewing her lips, the psychiatrist changed her medication to aripiprazole $10 \mathrm{mg} / \mathrm{dai}-$ ly. On the 4th day of aripiprazole treatment, she showed almost 
no response to verbal stimuli or light pain. As eating and relieving herself unaided were beyond the patient's abilities, a nasogastric tube was inserted. Since she had a mild fever, she underwent hematological tests and a chest X-ray, but these revealed no particular abnormal findings. Despite receiving continuous antipsychotic medication, the patient showed no improvement. Therefore, she was transferred to our university hospital for further evaluation.

Upon her admission, the patient's symptoms were continued. Moreover, as she showed intermittent rigidity, she gnawed her teeth and bit her lips, she lost her 4 teeth. We performed a brain magnetic resonance imaging (MRI), spinal fluid tap, and electroencephalogram (EEG), but the brain MRI and the spinal fluid revealed no particular findings. In EEGs of the patient, we observed a slow wave in the frontal lobe. Due to the patient's fever, we administered antibiotics, but the fever did not recede. Findings from the department of internal medicine showed no particular active lung lesion on the chest X-ray and suspicious lesion was focal consolidation finding which would be found on chest computer tomography (CT) only. Microorganism was not cultivated on the blood microorganism study and any other microorganism was not cultivated except normal flora on the sputum microorganism study. Consequently, because the lesion of the chest was small and minor, the medical doctor did not consider the lesion to be causing the patient's continuous high fever. Her blood pressure remained in a relatively normal range, and her electrocardiogram (ECG) did not show any particular findings except atrial tachycardia. On her $2^{\text {nd }}$ day in our hospital, her blood oxygen saturation suddenly dropped to $67 \%$, so we inserted a tracheal tube and placed her on a ventilator. The medical doctor believed a temporary laryngospasm had caused the oxygen saturation decrease. The reason of suspecting of laryngospasm was that the patient intermittently showed excessive motor activity, rigidity and, also dystonia on buccopharyngeal part, and that she did not have lesion that would lead to sudden dyspnea on chest X-ray and chest CT finding at that time. A chest CT scan did not show any particular change from the previous one. And considering that the patient did not have a history of asthma, it is highly possible that the patient has episodic laryngospasm. Despite continuous medication with antibiotics and antipyretics, her $38-39^{\circ} \mathrm{C}$ fever and 100-150 heart rate continued. In hematological tests, the patient's leukocyte count increased to 13,700, and her Creatine Phosphate Kinase (CPK) increased to 558. When we removed the respirator on her $14^{\text {th }}$ day in Intensive Care Unit (ICU), her respiration was stable, but the fever did not recede. On her $18^{\text {th }}$ day in ICU, the fever still continued, but the medical doctor judged that no further medical treatment was necessary. Therefore, the patient was transferred to the Psychiatric Ward.

On her 1st day in this ward, the patient stayed in the bed all day and showed only non-specific responses to verbal stimuli. She could not respond appropriately to questions, and her body became rigid or writhed several times a day. In hematological tests, her leukocyte count decreased to 11,940 than before, CPK also decreased to 341 than before, aspartase aminotransferase/ alanine aminotransferase ratio to 61/76, and serum iron level decreased to $30 \mu \mathrm{g} / \mathrm{dL}$. On her 9th day in the Psychiatric Ward, the patient's fever fell, and her heart rate became stable, so we decided to administer ECT treatment. After 3rd ECT treatments, the patient could have a short conversation with her family. For maintenance treatment, the patient received olanzapine (zyprexa zydis) $5 \mathrm{mg} /$ daily, which melts on the tongue, from the 5 th ECT. As she could speak her thought and feelings, she reported that she had some psychotic symptoms such as auditory hallucination, delusion of possession before admission in the psychiatric hospital. The patient stated she had been arrested by an evil spirit, or an evil spirit entered her at that time. After the 10th ECT, the patient could get up, walk, and do daily routines little by little. The patient underwent ECT total 16 times, and after two months of inpatient treatment, the patient did not show any psychotic symptoms. She has currently been receiving outpatient treatment for 9 months without complaining of any psychotic symptoms.

\section{DISCUSSION}

Diagnostic and Statistical Manual of Mental Disorders, $4^{\text {th }}$ edition, Text Revision (DSM-IV-TR) classifies catatonia into the sub-groups of schizophrenia, bipolar disorder, major depressive disorder, and general medical condition. ${ }^{11}$ Several recent studies, however, point out an association between catatonia and both neuroleptic use and pervasive developmental disorder. ${ }^{12}$ The researchers attempt particularly to define catatonia due to neuroleptics by first classifying the causes of catatonia as spontaneous, psychogenic, or neuroleptic. ${ }^{13,14}$ For this case, the symptoms of catatonia were not apparent at the onset of symptoms, but, rather, they developed rapidly after she received an antipsychotic drug. Any medicine except atypical antipsychotics was not used to the patient in the psychiatric hospital and our university hospital, and only lorazepam 2-4 mg intramuscular injection was intermittently used to improve her rigidity and irritability. In this case, we cannot assert definitely that the use of an antipsychotic drug caused her catatonia, but we at least can assume that the antipsychotic drug may be associated with her catatonia. Our patient showed the catatonia symptoms of extreme mutism, rigidity, negativism, and stereotyped movement after using antipsychotic drug, and later showed mental stupor and a high fever, leading us to suspect NMS. According to recent reports, NMS due to an atypical antipsychotic drug differs from the characteristic symptoms of NMS due to a typi- 
cal antipsychotic drug, in that it occurs acutely, within a few days of the patient beginning the medication, and may show an atypical symptom pattern, for the muscle rigidity, consciousness alteration, and CPK increase do not appear clearly. ${ }^{15-20}$ For this reason, Nopoulos et al. ${ }^{21}$ suggested that this should be called "atypical NMS" or "pseudo-neuroleptic malignant syndrome," to distinguish it. There have already been many case reports on patients developing NMS from lethal catatonia, ${ }^{22}$ and a number of studies reported similarities between NMS and catatonia in terms of their clinical characteristics, ${ }^{23,24}$ biological characteristics, ${ }^{7,25-28}$ and treatment response patterns. ${ }^{29-32}$ In particular, many authors maintain that lethal catatonia cannot be clinically separated from NMS. ${ }^{8,23,24}$ With regard to treatment responses, $40 \%$ of NMS patients responded to benzodiazepine, ${ }^{33}$ and Lee's study classified NMS patients into either a catatonic type, which showed catatonia symptoms and responded to benzodiazepine during the NMS episode, and a non-catatonic type that did not do so.2 Accordingly, some researchers regard catatonia that responds to benzodiazepine as an NMS subtype. ${ }^{31}$

Due to such characteristics, some researchers consider catatonia and NMS to be disorders along a single spectrum,, 23 and there are even such reports as that of Tsai et al., ${ }^{14}$ stating NMS and catatonia are in the same "neuroleptic toxicity spectrum" category, and of Mathews and Aderibigbe, regarding NMS as a severe subtype of catatonia, classified as "drug-induced hyperthermic catatonia." ${ }^{34}$ And some researchers regarded catatonia, extrapyramidal symptoms (EPS), agitation, and low serum iron level as risk factors of NMS. ${ }^{2,25,35}$

Summing up, the case in this study does not constitute clear evidence that antipsychotic drugs are a direct cause of catatonia symptoms, but, inferring from previous reports, we can at least presume antipsychotic drugs are associated with catatonia. Furthermore, regarding the NMS occurring after the catatonia, we observed symptoms similar to recent case reports of NMS caused by an atypical antipsychotic drug. In our case, ECT made the great contribution to the patient's rapid improvement. Given that, reported, morbidity and mortality may increase if catatonia is not treated early, ${ }^{36}$ ECT may be an effective treatment for a patient who does not respond to the usual medications, depending on the patient's condition.

\section{REFERENCES}

1. Fink M. Treating neuroleptic malignant syndrome as catatonia. J Clin Psychopharmacol 2001;21:121-122.

2. Paparrigopoulos T, Tzavellas E, Ferentinos P, Mourikis I, Liappas J. Catatonia as a risk factor for the development of neuroleptic malignant syndrome: report of a case following treatment with clozapine. World J Biol Psychiatry 2009;10:70-73.

3. Peralta V, Cuesta MJ, Serrano JF, Mata I. The Kahlbaum syndrome: a study of its clinical validity, nosological status, and relationship with schizophrenia and mood disorder. Compr Psychiatry 1997;38:61-67.

4. Northoff G. What catatonia can tell us about "top-down modulation": a neuropsychiatric hypothesis. Behav Brain Sci 2002;25:555-577.

5. Caroff SN, Mann SC. Neuroleptic malignant syndrome. Med Clin North Am 1993;77:185-202.

6. Hasan S, Buckley P. Novel antipsychotics and the neuroleptic malignant syndrome: a review and critique. Am J Psychiatry 1998;155:11131116.

7. Mann SC, Caroff SN, Fricchione G, Campbell EC. Central dopamine hypoactivity and the pathogenesis of the neuroleptic malignant syndrome. Psychiatr Ann 2000;30:363-374.

8. White DA. Catatonia and the neuroleptic malignant syndrome--a single entity? Br J Psychiatry 1992;161:558-560.

9. White DA, Robins AH. Catatonia: harbinger of the neuroleptic malignant syndrome. Br J Psychiatry 1991;158:419-421.

10. Raja M, Altavista MC, Cavallari S, Lubich L. Neuroleptic malignant syndrome and catatonia. A report of three cases. Eur Arch Psychiatry Clin Neurosci 1994;243:299-303.

11. American Psychiatric Association. Diagnostic and Statistical Manual of Mental Disorders. Fourth Edition. Text Revision. Washington, DC: American Psychiatric Press; 2000.

12. Dhossche DM, Carroll BT, Carroll TD. Is there a Common Neuronal Basis for Autism and Catatonia? In: Dhossche D, Wing L, Ohta M, Neumarker K, Editors. Catatonia in Autism Spectrum Disorders. St. Louis, MO: Elsevier/Academic Press, Inc; 2006.

13. Woodbury MM, Woodbury MA. Neuroleptic-induced catatonia as a stage in the progression toward neuroleptic malignant syndrome. J Am Acad Child Adolesc Psychiatry 1992;31:1161-1164.

14. Tsai JH, Yang P, Yen JY, Chen CC, Yang MJ. Zotepine-induced catatonia as a precursor in the progression to neuroleptic malignant syndrome. Pharmacotherapy 2005;25:1156-1159.

15. Chakraborty N, Johnston T. Aripiprazole and neuroleptic malignant syndrome. Int Clin Psychopharmacol 2004;19:351-353.

16. Leibold J, Patel V, Hasan RA. Neuroleptic malignant syndrome associated with ziprasidone in an adolescent. Clin Ther 2004;26:1105-1108.

17. Zalsman G, Lewis R, Konas S, Loebstein O, Goldberg P, Burguillo F, et al. Atypical neuroleptic malignant syndrome associated with risperidone treatment in two adolescents. Int J Adolesc Med Health 2004; 16:179-182.

18. Dew RE, Rosenquist PB, McCall WV. Aripiprazole for agitation in a 13-year-old girl with neuroleptic malignant syndrome. Int J Adolesc Med Health 2005;17:187-188.

19. Kobayashi A, Kawanishi C, Matsumura T, Kato D, Furukawa R, Kishida I, et al. Quetiapine-induced neuroleptic malignant syndrome in dementia with Lewy bodies: a case report. Prog Neuropsychopharmacol Biol Psychiatry 2006;30:1170-1172.

20. Norgard NB, Stark JE. Olanzapine-associated neuroleptic malignant syndrome. Pharmacotherapy 2006;26:1180-1182.

21. Nopoulos P, Flaum M, Miller DD. Atypical neuroleptic malignant syndrome (NMS) with an atypical neuroleptic: clozapine-induced NMS without rigidity. Ann Clin Psychiatry 1990;2:251-253.

22. Lee JW. Catatonic variants, hyperthermic extrapyramidal reactions, and subtypes of neuroleptic malignant syndrome. Ann Clin Psychiatry 2007;19:9-16.

23. Fink M. Neuroleptic malignant syndrome and catatonia: one entity or two? Biol Psychiatry 1996;39:1-4.

24. Carroll BT, Taylor RE. The nondichotomy between lethal catatonia and neuroleptic malignant syndrome. J Clin Psychopharmacol 1997;17: 235-238.

25. Carroll BT, Goforth HW. Serum iron in catatonia. Biol Psychiatry 1995;38:776-777.

26. Nisijima K, Ishiguro T. Cerebrospinal fluid levels of monoamine metabolites and gamma-aminobutyric acid in neuroleptic malignant syndrome. J Psychiatr Res 1995;29:233-244.

27. Northoff G, Wenke J, Pflug B. Increase of serum creatine phosphokinase in catatonia: an investigation in 32 acute catatonic patients. Psy- 
chol Med 1996;26:547-553.

28. Northoff G, Pfennig A, Krug M, Danos P, Leschinger A, Schwarz A, et al. Delayed onset of late movement-related cortical potentials and abnormal response to lorazepam in catatonia. Schizophr Res 2000;44:193211.

29. Lew TY, Tollefson G. Chlorpromazine-induced neuroleptic malignant syndrome and its response to diazepam. Biol Psychiatry 1983;18:14411446.

30. Kontaxakis VP, Christodoulou GN, Markidis MP, Havaki-Kontaxaki BJ. Treatment of a mild form of neuroleptic malignant syndrome with oral diazepam. Acta Psychiatr Scand 1988;78:396-398.

31. Miyaoka H, Shishikura K, Otsubo T, Muramatsu D, Kamijima K. Diazepam-responsive neuroleptic malignant syndrome: a diagnostic subtype? Am J Psychiatry 1997;154:882.

32. Koch M, Chandragiri S, Rizvi S, Petrides G, Francis A. Catatonic signs in neuroleptic malignant syndrome. Compr Psychiatry 2000;41:73-75.

33. Addonizio G, Susman VL, Roth SD. Neuroleptic malignant syndrome: review and analysis of 115 cases. Biol Psychiatry 1987;22:1004-1020.

34. Mathews T, Aderibigbe YA. Proposed research diagnostic criteria for neuroleptic malignant syndrome. Int J Neuropsychopharmacol 1999; 2:129-144.

35. Carroll BT, Lee JW. Catatonia is a risk factor for neuroleptic malignant syndrome. J Clin Psychiatry 2004;65:1722-1723.

36. Malur C, Pasol E, Francis A. ECT for prolonged catatonia. J ECT 2001; 17:55-59. 